\title{
NUMERICAL SIMULATION OF INCOMPRESSIBLE FLOWS BY THE STABILIZED FINITE ELEMENT METHOD
}

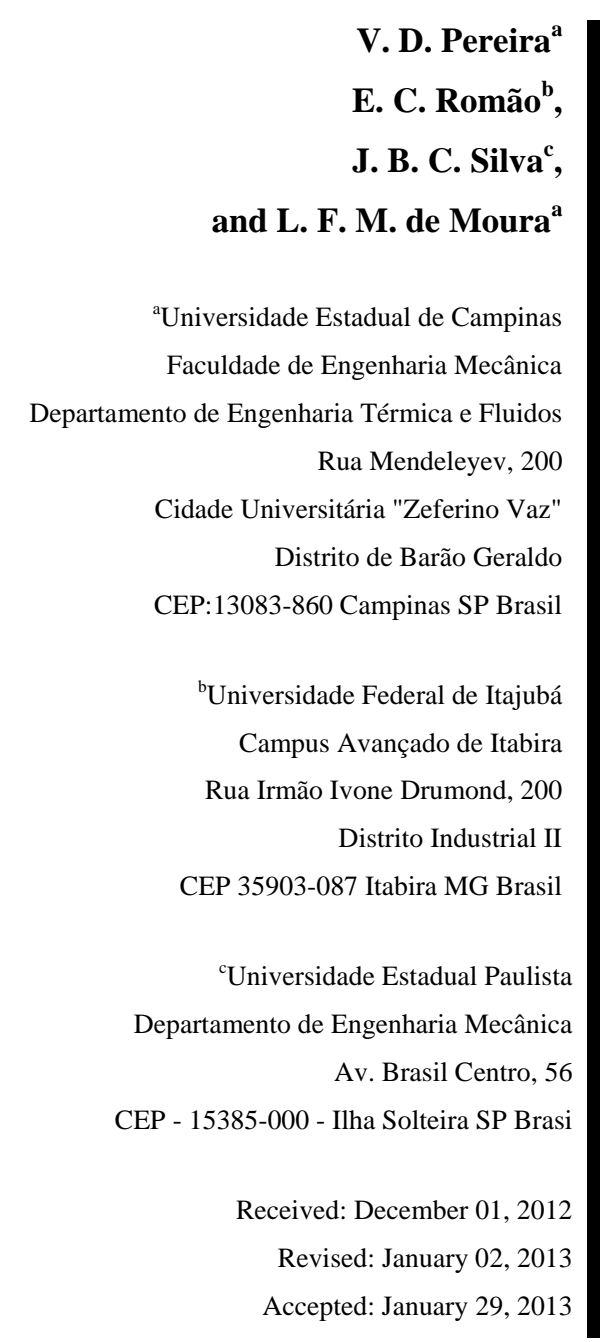

NOMENCLATURE

$N \quad$ interpolation function

Pr Prandt number

Re Reynolds number

$t \quad$ time coordinate

$T$ temperature

$u_{i} \quad$ velocity field

$x_{i} \quad$ space coordinates

\section{Greek symbols}

$\Omega \quad$ two-dimensional domain

$\alpha \quad$ diffusion coefficient

$\rho \quad$ especific mass

\section{ABSTRACT}

The fast progress has been observed in the development of numerical and analytical techniques for solving convection-diffusion and fluid mechanics problems. Here, a numerical approach, based in Galerkin Finite Element Method with Finite Difference Method is presented for the solution of a class of non-linear transient convection-diffusion problems. Using the analytical solutions and the $L_{2}$ and $L_{\infty}$ error norms, some applications is carried and valuated with the literature.

Keywords: Numerical simulation, Burgers equation, Galerkin Finite Element Method, Finite Difference Method, Cranck-Nicolson Method.

\section{INTRODUCTION}

The calculations of fluid flow are of interest in many processes of importance to man and also in nature. Such flows are modeled mathematically by the Navier Stokes equations, which are nonlinear partial differential equations. These equations are very difficult to solve analytically, except in very simplified cases.

Due to the nonlinearities and complicated geometries found in real problems, solutions of the Navier-Stokes equations, in its complete form, are only possible by numerical methods. The finite element method (FEM) is based on the weighted residuals method (WRM), which gives rise to different formulations: Bubnov-Galerkin, PetrovGalerkin, Placement, Subdomain and Least Squares. These formulations result of the choice of the weight function in inner product of the residue by the weight function, in the integral or variational formulation of the method. 
The current literature on the finite element method is vast, with emphasis of the textbooks presented by Zienkiewicz and Taylor (2005), Reddy (1993) and Jiang (1998). Jiang (1998) presents the detailed formulation of the least squares finite element method (LSFEM) for computational fluid dynamics and electromagnetism.

It is well known that the classical Galerkin method produces oscillations solving convective dominant problems. However, this method is great for purely diffusive problems. According to Lewis et al. (2004), the Characteristic Based Split method has been used for solving convection-diffusion equations, initially for the case of compressible flows and more recently for the case of incompressible flows. In this case, when considering the motion of a particle in the direction of the characteristic, a convection-diffusion equation is transformed into a diffusion equation and the classical Galerkin method can be applied without oscillations in the solution. This alternative of stabilizing the finite element method is also used in this paper for solving the Navier-Stokes equations with heat transfer, for some two-dimensional problems, by using linear triangular elements.

The stabilization method used in this work was a method of discretization of the time derivative term based on the flow along the characteristic, called in the literature Characteristic Based Split (CBS) scheme. The CBS scheme was originally presented by Zienkiewicz and Taylor (2005). Excellent works on this method are Lewis et al. (2004) and Liu (2005).

Liu (2005) presented a methodology CBS with artificial compressibility (AC) and a semi-implicit CBS scheme for incompressible laminar and turbulent flows. The numerical simulations for steady-state and transient incompressible flows were held in structured and unstructured meshes of linear triangular and tetrahedral elements. The standard Galerkin method was used for spatial discretization of governing equations, with a semi- implicit CBS. The mathematical model was based on Navier-Stokes averaged equations (RANS) and four turbulence models of two equations were studied in detail. Results for laminar and turbulent flows, in unsteady and steady-state, were obtained. In addition to problems of the steady-state flow, the unsteady-state averaged Navier-Stokes (URANS) model was used to simulate the vortices behind a circular cylinder using the technique of dual time step. Two and threedimensional results have shown that both the CBSAC procedure (matrix free) and the semi-implicit CBS formulation are accurate and efficient.

\section{MATHEMATICAL MODEL}

Fluid flows can be modeled mathematically by the Navier Stokes equations, which are nonlinear partial differential equations. The dimensionless form of these equations is shown below.

$$
\begin{gathered}
\frac{\partial U_{i}}{\partial t}+U_{j} \frac{\partial U_{i}}{\partial X_{i}}+\frac{\partial P_{i}}{\partial X_{i}}+\varepsilon_{i j k}\left(\frac{1}{\operatorname{Re}}+v_{t}\right) \frac{\partial \Omega_{k}}{\partial X_{i}}=S_{i} \\
\frac{\partial U_{i}}{\partial X_{i}}=0 \\
\Omega_{i}=\varepsilon_{i j k} \frac{\partial U_{k}}{\partial X_{j}} \\
\frac{\partial \Omega_{i}}{\partial X_{i}}=0
\end{gathered}
$$

\section{NUMERICAL MODEL: SEPARATION MODEL BASED ON THE CHARACTERISTIC}

The separation model based on the characteristic is used to stabilize the classical Galerkin method, since this method presents oscillating solutions when applied to the solution of convection dominant problems. The convectiondiffusion equation for a scalar variable $\phi$ in the not conservative form is:

$$
\frac{\partial \phi}{\partial t}+u_{i} \frac{\partial \phi}{\partial x_{i}}-\frac{\partial}{\partial x_{i}}\left(\alpha \frac{\partial \phi}{\partial x_{i}}\right)+Q=0
$$

where $u_{i}$ is the velocity field, $\phi$ is the property transported by convection and diffusion, $\alpha$ is the diffusion coefficient and $Q$ is any external source of $\phi$.

In Eq. (5), if the linear convection term is considered one-dimensional with constant velocity, then the characteristic propagates in the plane $(\phi, t)$ as shown in Fig. 1. Thus, we can write:

$$
\phi\left(\hat{x}+\delta, t_{n+1}\right)-\phi\left(\hat{x}, t_{n}\right)=0
$$

where $\delta$ is the distance traveled by the particle with a characteristic velocity equal to the convective velocity $u$ and $\hat{x}=x-u d t$ is the one-dimensional characteristic direction.

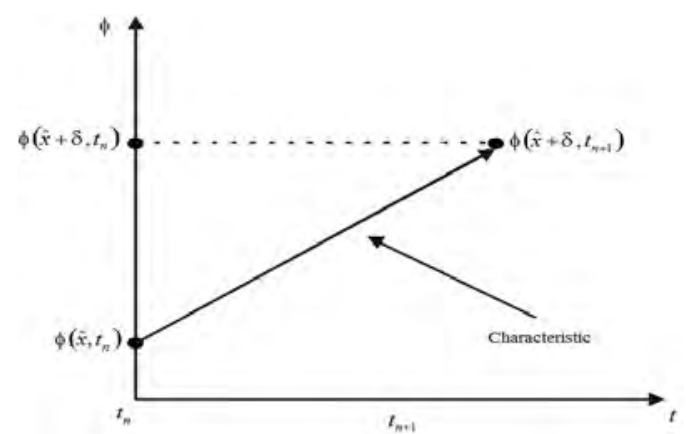

Figure 1. Linear characteristic scheme.

Equation (6) can be integrated, in the form of weighted residue, using the weight function $w(\hat{x}+\delta)$ : 


$$
\int_{\Omega} w(\hat{x}+\delta)\left[\phi\left(\hat{x}+\delta, t_{n+1}\right)-\phi\left(\hat{x}, t_{n}\right)\right] d \Omega=0
$$

With the substitution of the interpolation functions, Equation (7) becomes:

$$
\begin{aligned}
\int_{\Omega} N^{j}(\hat{x}+\delta)\left[N^{j}(\hat{x}+\delta) \phi\left(\hat{x}+\delta, t_{n+1}\right)-\right. & \\
& \left.-N^{i}(\hat{x}) \phi\left(x, t_{n}\right)\right] d \Omega=0
\end{aligned}
$$

The exact integration of Eq. (8) is not possible. Then, an approximate integration procedure must be used. The splitting procedure used is the semiimplicit CBS scheme, based on Zienkiewicz and Codina (1995). A dimensionless form of Eq. (5) can be obtained by using the following scales:

$u_{i}^{*}=\frac{u_{i}}{u_{\infty}} ; \rho^{*}=\frac{\rho}{\rho_{\infty}} ; \mu^{*}=\frac{\mu}{\mu_{\infty}} ; \alpha^{*}=\frac{\alpha}{\alpha_{\infty}} x_{i}^{*}=\frac{x_{i}}{L} ;$

$t^{*}=\frac{t u_{\infty}}{L} ; p^{*}=\frac{p}{\rho_{\infty} u_{\infty}^{2}} ; \tau_{i j}^{*}=\frac{\tau_{i j}}{\rho_{\infty} u_{\infty}^{2}} ; T^{*}=\frac{T-T_{\infty}}{T_{w}-T_{\infty}}$

where * indicates dimensionless quantities, the subscript $\infty$ represents the quantities in the free stream and $\mathrm{L}$ is a reference length.

The semi-implicit CBS scheme consists of three steps. In the first step, an intermediate velocity is calculated. In the second step, the pressure is obtained from the modified continuity equation. In the third step, the intermediate velocity is corrected to obtain the final velocity. From the velocity field, any scalar variable can be obtained by solving the transport equation as a fourth step. The Navier-Stokes equations and energy equation for the temperature, in dimensionless form, can be written as:

$$
\begin{gathered}
\frac{\partial U}{\partial t}+u_{j} \frac{\partial U_{i}}{\partial X_{j}}+\frac{\partial P}{\partial X_{i}}-\frac{1}{\operatorname{Re}} \frac{\partial \tau_{j i}}{\partial X_{j}}=S_{i} \\
\frac{\partial U_{i}}{\partial X_{i}}=0 \\
\frac{\partial T}{\partial t}+u_{j} \frac{\partial T}{\partial X_{j}}-\frac{1}{\operatorname{Re} \operatorname{Pr}} \frac{\partial}{\partial X_{j}}\left(\alpha \frac{\partial T}{\partial X_{j}}\right)=Q
\end{gathered}
$$

According to Liu (2005), the four time steps in the discretization of Eqs. (10) to (12), using the semiimplicit CBS scheme, are:

Step 1 - Intermediate momentum

$\Delta U_{j}^{*}=U_{j}^{*}-U_{j}^{n}=\Delta t\left[-\frac{\partial}{\partial x_{k}}\left(u_{k} U_{j}\right)+\frac{1}{\operatorname{Re}} \frac{\partial \tau_{i j}}{\partial x_{i}}\right]^{n}+$
$+\frac{(\Delta t)^{2}}{2}\left\{u_{m} \frac{\partial}{\partial x_{m}}\left[\frac{\partial\left(u_{k} U_{j}\right)}{\partial x_{k}}-\frac{1}{\operatorname{Re}} \frac{\partial \tau_{i j}}{\partial x_{i}}\right]\right\}^{n}$

where $U_{j}^{n}=U_{j}\left(t_{n}\right)=\rho u_{j}^{n} \quad$ is the fluid particle momentum per unit volume, * indicates an intermediate quantity, $\Delta t=t^{n+1}-t^{n}$, and $\operatorname{Re}=\frac{\rho_{\infty} u_{\infty} L}{\mu_{\infty}}$ is the Reynolds number.

Step 2 - Pressure

$\left(\frac{1}{c^{2}}\right)^{n} \Delta p=\left(\frac{1}{c^{2}}\right)^{n}\left(p^{n+1}-p^{n}\right)=-\Delta t\left[\frac{\partial U_{j}^{n}}{\partial x_{j}}+\right.$
$\left.+\theta_{1} \frac{\partial \Delta U_{j}^{*}}{\partial x_{j}}-\Delta t \theta_{1}\left(\frac{\partial^{2} p^{n}}{\partial x_{j} \partial x_{j}}+\theta_{2} \frac{\partial^{2} \Delta p}{\partial x_{j} \partial x_{j}}\right)\right]$

where $c$ is the speed of sound. In Eq. (14) it is assumed that density changes are related to pressure changes, for small compressibility or elastic deformation, and $c$ approaches infinity for incompressible flows.

Step 3 - Momentum correction

$$
\Delta U_{j}=U_{j}^{n+1}-U_{j}^{n}=\Delta U_{j}^{*}-\Delta t \frac{\partial p^{n+\theta_{2}}}{\partial x_{j}}
$$

where $0,5 \leq \theta_{1} \leq 1$ and $\theta_{2}=0$ for the explicit formulation and $0,5 \leq \theta_{1} \leq 1$ and $0,5 \leq \theta_{2} \leq 1$ for the semi-implicit formulation.

Step 4 - Energy equation

$$
\begin{aligned}
& \Delta T=T\left(x_{i}, t_{n+1}\right)-T\left(x_{i}, t_{n}\right)=\Delta t\left[-u_{j}\left(x_{j}, t_{n}\right) .\right. \\
& \left.\frac{\partial T\left(x_{j}, t_{n}\right)}{\partial x_{j}}+\frac{1}{\operatorname{Re} \operatorname{Pr}} \frac{\partial}{\partial x_{i}}\left(\alpha \frac{\partial T\left(x_{i}, t_{n}\right)}{\partial x_{i}}\right)-Q\left(x_{i}, t_{n}\right)\right]+ \\
& +\frac{(\Delta t)^{2}}{2}\left\{u_{i}\left(x_{i}, t_{n}\right) \frac{\partial}{\partial x_{i}}\left(u_{j}\left(x_{j}, t_{n}\right) \frac{\partial T\left(x_{j}, t_{n}\right)}{\partial x_{j}}\right)\right\}+ \\
& \frac{(\Delta t)^{2}}{2}\left\{-u_{j}\left(x_{j}, t_{n}\right) \frac{1}{\operatorname{Re} \operatorname{Pr}} \frac{\partial}{\partial x_{j}}\left[\frac{\partial}{\partial x_{i}}\left(\alpha \frac{\partial T\left(x_{j}, t_{n}\right)}{\partial x_{i}}\right)\right]+\right. \\
& \left.u_{j}\left(x_{j}, t_{n}\right) \frac{\partial Q\left(x_{j}, t_{n}\right)}{\partial x_{j}}\right\}
\end{aligned}
$$

The second order extra terms in the last part of the right side in steps 1 and 4 are consistent and reduce the oscillations due to the typical Galerkin discretization for the convective terms. The third or higher order terms disappear when linear elements are employed. The boundary conditions for the CBS scheme are the first kind (Dirichlet) or the second kind (Neumann). The Dirichlet boundary conditions for velocity, like no slip conditions, are imposed in 
step 3. The traction boundary conditions are prescribed in step 1. No Dirichlet condition for pressure is essential for the explicit CBS scheme, but at least one pressure boundary condition is essential for the semi implicit scheme.

The following spatial discretization by the Galerkin method is employed:

$U_{j}=N_{u} \tilde{U}_{j} ; \Delta U_{j}=N_{u} \Delta \tilde{U}_{j} ; \Delta U_{j}^{*}=N_{u} \Delta \tilde{U}_{j}^{*} ;$

$u_{j}=N_{u} \tilde{u}_{j} ; \Delta p=N_{p} \Delta \tilde{p} ; T=N_{T} \tilde{T}$

where $N$ are the interpolation functions and the tilde indicates a nodal quantity, i.e.:

$$
\begin{aligned}
\tilde{U}_{j} & =\left[\begin{array}{llllll}
U_{j}^{1} & U_{j}^{2} & \cdots & U_{j}^{k} & \cdots & U_{j}^{l}
\end{array}\right]^{T} \\
N & =\left[\begin{array}{llllll}
N^{1} & N^{2} & \cdots & N^{k} & \cdots & N^{l}
\end{array}\right]
\end{aligned}
$$

Applying the Galerkin approximation to the divergence theorem, one arrives at the weak formulation of the governing equations as follows.

Step 1 - Weak formulation of intermediary momentum

$$
\begin{array}{r}
\int_{\Omega} N_{u}^{T} \Delta U_{j}^{*} d \Omega=\Delta t\left[-\int_{\Omega} N_{u}^{T} \frac{\partial\left(u_{k} U_{j}\right)}{\partial x_{k}} d \Omega-\right. \\
\left.-\frac{1}{\operatorname{Re}} \int_{\Omega} \frac{\partial N_{u}^{T}}{\partial x_{i}} \tau_{i j} d \Omega\right]^{n}+\frac{\Delta t^{2}}{2}\left[\int_{\Omega} \frac{\partial\left(u_{m} N_{u}^{T}\right)}{\partial x_{m}} .\right. \\
\left.\cdot\left(-\frac{\partial\left(u_{k} U_{j}\right)}{\partial x_{k}}\right) d \Omega\right]^{n}+\Delta t\left[\int_{\Gamma} N_{u}^{T} t_{d} d \Gamma\right]^{n}
\end{array}
$$

In Eq. (20), $t_{d}=\left(\tau_{i j} / \mathrm{Re}\right) n$ indicates the portion of the traction corresponding to the normal stress and $n$ is the normal vector outward of the boundary.

Step 2 - Weak formulation of pressure equation

$$
\begin{gathered}
\int_{\Omega} N_{p}^{T}\left(\frac{1}{\beta^{2}}\right)^{n} \Delta p d \Omega=-\Delta t \int_{\Omega} N_{p}^{T} \frac{\partial}{\partial x_{j}} U_{j}^{n} d \Omega- \\
-\Delta t \int_{\Gamma} N_{p}^{T}\left(\Delta U_{j}^{*}-\Delta t \frac{\partial p^{n}}{\partial x_{j}}\right) n_{j} d \Gamma+ \\
+\Delta t \int_{\Omega} \frac{\partial N_{p}^{T}}{\partial x_{j}}\left(\Delta U_{j}^{*}-\Delta t \frac{\partial p^{n}}{\partial x_{j}}\right) d \Omega
\end{gathered}
$$

In Eq. (21) the pressure and the term $\Delta U_{j}^{*}$ are integrated by parts and $n_{j}$ are the components of the normal vector outward of the boundary.

The semi-implicit scheme is:

$$
\begin{gathered}
\int_{\Gamma} N_{p}^{T} \frac{\partial p^{n+1}}{\partial x_{j}} n_{j} d \Gamma-\int_{\Gamma} \frac{\partial N_{p}^{T}}{\partial x_{j}} \frac{\partial p^{n+1}}{\partial x_{j}} d \Omega= \\
=\frac{1}{\Delta t} \int_{\Omega} N_{p}^{T} \frac{\partial U_{j}^{*}}{\partial x_{j}} d \Omega
\end{gathered}
$$

In Eq. (22), the pressure term is integrated by parts.

Step 3 - Weak formulation of the momentum correction

$$
\begin{aligned}
\int_{\Omega} N_{u}^{T} \Delta U_{j} d \Omega & =\int_{\Omega} N_{u}^{T} \Delta U_{j}^{*} d \Omega+ \\
& +\Delta t \int_{\Omega} \frac{\partial N_{u}^{T}}{\partial x_{j}} p^{n} d \Omega-\Delta t \int_{\Gamma} N_{u}^{T} t_{p} d \Gamma
\end{aligned}
$$

The semi-implicit scheme is:

$$
\begin{aligned}
\int_{\Omega} N_{u}^{T} & \Delta U_{j} d \Omega=\int_{\Omega} N_{u}^{T} \Delta U_{j}^{*} d \Omega+ \\
& +\Delta t \int_{\Omega} \frac{\partial N_{u}^{T}}{\partial x_{j}} p^{n+1} d \Omega-\Delta t \int_{\Gamma} N_{u}^{T} t_{p} d \Gamma
\end{aligned}
$$

In Eqs. (23) and (24), $t_{p}=\left(p^{n}+\theta_{2} \Delta p\right) n$ only indicates the portion of the traction corresponding to the pressure which was removed in step 1 . It is simply ignored and assumed to be zero since complete traction is prescribed in step 1.

Step 4 - Weak formulation of the energy equation

$$
\begin{gathered}
\int_{\Omega} N_{T}^{T} \Delta T d \Omega=\Delta t\left[-\int_{\Omega} N_{T}^{T} \frac{\partial\left(u_{k} T\right)}{\partial x_{k}} d \Omega-\right. \\
\left.-\frac{1}{\operatorname{Re} \operatorname{Pr}} \int_{\Omega} \frac{\partial N_{T}^{T}}{\partial x_{i}} \alpha \frac{\partial T}{\partial x_{i}} d \Omega\right]^{n}+\frac{\Delta t^{2}}{2} . \\
\cdot\left[\int_{\Omega} \frac{\partial\left(u_{m} N_{T}^{T}\right)}{\partial x_{m}}\left(-\frac{\partial\left(u_{k} T\right)}{\partial x_{k}}\right) d \Omega\right]^{n}+ \\
+\Delta t \frac{1}{\operatorname{Re} \operatorname{Pr}}\left[\int_{\Gamma} N_{T}^{T} \alpha \frac{\partial T}{\partial x_{i}} n_{i} d \Gamma\right]^{n}
\end{gathered}
$$

The final forms of the matrices in the weak formulation of the governing equations are:

Step 1 - Intermediary momentum

$$
\Delta \tilde{U}^{*}=-M_{u}^{-1} \Delta t\left[\left(C_{u} \tilde{U}+K_{\tau} \tilde{u}-f_{u}\right)-\Delta t\left(K_{u} \tilde{U}\right)\right]^{n}
$$

Step 2 - Pressure 


$$
\begin{aligned}
&\left(M_{p}+\Delta t^{2} \theta_{1} \theta_{2} H\right) \Delta \tilde{p}= \\
& \Delta t\left[\left(G \tilde{U}+K_{\tau} \tilde{u}-f_{u}\right)-\Delta t\left(K_{u} \tilde{U}\right)\right]^{n}
\end{aligned}
$$

Step 3 - Momentum correction

$$
\Delta \tilde{U}=\Delta \tilde{U}^{*}-M_{u}^{-1} \Delta t\left[G^{T}\left(\tilde{p}^{n}+\theta_{2} \Delta \tilde{p}\right)\right]
$$

Step 4 - Energy equation

$$
\Delta \widetilde{T}=-M_{T}^{-1} \Delta t\left[C_{T} \tilde{T}+K_{t} \tilde{T}+K_{s} \tilde{T}-f_{T}\right]^{n}
$$

where:

$$
\begin{aligned}
& M_{u}=\int_{\Omega} N_{u}^{T} N_{u} d \Omega ; \\
& K_{\tau}=\int_{\Omega} B^{T} \frac{(\rho v)}{\operatorname{Re}}\left(I_{0}-\frac{2}{3} m m^{T}\right) B d \Omega ; \\
& H=\int_{\Omega}\left(\nabla N_{p}\right)^{T} \nabla N_{p} d \Omega ; \\
& M_{p}=\int_{\Omega} N_{p}^{T}\left(\frac{1}{\beta^{2}}\right)^{n} N_{p} d \Omega ; \\
& f_{p}=\Delta t \int_{\Gamma} N_{p}^{T}\left[N_{u} \tilde{U}^{n}+\theta_{1}\left(\Delta \tilde{U}^{*}-\Delta t \nabla p^{n+\theta_{2}}\right)\right] n^{T} d \Gamma ; \\
& K_{u}=-\frac{1}{2} \int_{\Omega}\left(\nabla^{T}\left(u N_{u}\right)\right)^{T}\left(\nabla^{T}\left(u N_{u}\right)\right) d \Omega ; \\
& G=\int_{\Omega}\left(\nabla N_{p}\right)^{T} N_{u} d \Omega ; \\
& f_{u}=\int_{\Gamma} N_{u}^{T} t_{d} d \Gamma ; \\
& C_{u}=\int_{\Omega} N_{u}^{T}\left(\nabla^{T}\left(u N_{u}\right)\right) d \Omega ; \\
& M_{T}=\int_{\Omega} N_{T}^{T} N_{T} d \Omega ; \\
& C_{T}=\int_{\Omega} N_{T}^{T}\left(\nabla^{T}\left(u N_{T}\right)\right) d \Omega ; \\
& K_{T}=-\frac{1}{2} \int_{\Omega}\left(\nabla^{T}\left(u N_{u}\right)\right)^{T}\left(\nabla^{T}\left(u N_{u}\right)\right) d \Omega ; \\
& K_{s}=\frac{1}{\operatorname{RePr}} \int_{\Omega}\left(\nabla N_{T}\right)^{T} \alpha \nabla N_{T} d \Omega ; \\
& f_{T}=\frac{1}{\operatorname{Re} \operatorname{Pr}} \int_{\Gamma} N_{u}^{T} q d \Gamma
\end{aligned}
$$

The matrix of the strain function $B$ is given as:

$$
B=S N_{u}
$$

where $\boldsymbol{S}$ is an operator of tension matrix. For the twodimensional case, this operator is defined as:

$$
\begin{aligned}
& S=\left\{\begin{array}{cc}
\frac{\partial}{\partial x_{1}} & 0 \\
0 & \frac{\partial}{\partial x_{2}} \\
\frac{\partial}{\partial x_{2}} & \frac{\partial}{\partial x_{1}}
\end{array}\right\} \\
& m=\left[\begin{array}{lll}
1 & 1 & 0
\end{array}\right]^{T} \\
& I_{o}=\left[\begin{array}{lll}
2 & & \\
& 2 & \\
& & 1
\end{array}\right]
\end{aligned}
$$

In many problems of interest, the density remains approximately constant. This behavior is called incompressibility. An incompressible flow is generally defined using both parameters, velocity and pressure. Mixed formulations are often employed in finite element methods. Most forms of mixed Galerkin method results in discrete equations, which can usually be written as follows:

$$
\left[\begin{array}{cc}
K & G^{T} \\
G & M
\end{array}\right]\left[\begin{array}{l}
\tilde{u} \\
\tilde{p}
\end{array}\right]=\left[\begin{array}{l}
f_{1} \\
f_{2}
\end{array}\right]
$$

where $\tilde{u}$ is the primary discrete variable and $\tilde{p}$ is the discrete constraint variable (equivalent to the Lagrangean multiplier). The matrix $G$ is the discrete gradient operator, $K$ and $M$ are symmetric square matrices $n \times n$. $K$ is positive definite and $M$ is negative definite or zero, depending on the type of discretization used. $f_{1}$ and $f_{2}$ are source terms.

This section presents the way to avoid the LBB stability constraint that makes that, in the case of zero mass matrix $(\mathrm{M}=0)$, several useful elements cannot be used, since they result in oscillations of the pressure field and consequent non-convergence of the velocity field. For Stokes flow, Eq. (26) in step one only keeps the viscosity term and the term of traction at the boundary:

$$
\Delta \tilde{U}^{*}=-M_{u}^{-1} \Delta t_{t e m p}\left[K_{\tau} \tilde{u}^{n}-f_{u}\right]^{n}
$$

where the time step $\Delta t_{\text {temp }}$ provides temporal stability.

In step 2, the matrix $M_{p}$ disappears for incompressible flow and $\Delta \tilde{p}$ is equal to zero for steady state flow. Thus, Eq. (36) can be rewritten as:

$$
G \tilde{U}^{n}+\theta_{1} G \Delta \tilde{U}^{*}-\Delta t_{\text {spat }} \theta_{1} H \tilde{p}^{n}=f_{p}
$$


where spatial stability in discrete form is provided by time step $\Delta t_{\text {spat }}=l \Delta t_{\text {temp }}$, where $l$ is the ratio of time step. In steady state $\Delta \tilde{U}=0$, resulting in Eq. (28) in step 3 as:

$$
\Delta \tilde{U}^{*}=M_{u}^{-1} \Delta t_{\text {temp }}\left[G^{T} \tilde{p}^{n}\right]
$$

Therefore, the discretization results in the following matrix form:

$$
\left[\begin{array}{cc}
K_{v} & G^{T} \\
G & \Delta t_{\text {temp }} \theta_{1}\left(G M_{u}^{-1} G^{T}-l H\right)
\end{array}\right]\left[\begin{array}{c}
\tilde{U}^{n} \\
\tilde{p}^{n}
\end{array}\right]=\left[\begin{array}{c}
f_{u} \\
f_{p}
\end{array}\right]
$$

where the matrix $K_{v}=K_{\tau} / \rho$ is the quadratic form. The discrete velocity vector is $\tilde{U}^{n}$ and $\tilde{p}^{n}$ is the discrete vector of the nodal pressure.

If the approximate pressure is assumed to be discontinuous, then:

$$
\begin{aligned}
\tilde{p}^{n}= & \left(\Delta t_{\text {temp }} \theta_{1}\right)^{-1}\left(G M_{u}^{-1} G^{T}-l H\right)^{-1} f_{p}- \\
& \left(\Delta t_{\text {temp }} \theta_{1}\right)^{-1}\left(G M_{u}^{-1} G^{T}-l H\right)^{-1} G \tilde{U}^{n}
\end{aligned}
$$

and the system for $\tilde{U}^{n}$ is obtained by elimination of $\tilde{p}^{n}$. One can write:

$$
\left\lfloor K_{v}+\Psi G^{T} G\right\rfloor \tilde{U}^{n}=f_{u}+\Psi G^{T} f_{p}
$$

where the penalty function $\Psi=-1 /\left(\Delta t_{\text {temp }} \theta_{1} E\right)$ in witch $E=G M_{u}^{-1} G^{T}-l H$ is proportional to $\Delta t_{\text {temp }}$.

From physical considerations, one can show that the bilinear form $K_{v}$ is symmetric and positive definite. And from the quadratic form, one can show that $E$ is symmetric and negative definite. However, the system is always positive definite and leads to a nonsingular solution to $\tilde{U}^{n}$. Note that the discrete system, Eq. (39), has no zero diagonal term, so the restriction of LBB no longer influences the space of finite elements for velocity and pressure. So the system theoretically allows convenient and arbitrary interpolation functions for $\tilde{U}^{n}$ and $\tilde{p}^{n}$.

The criterion for evaluating the convergence to steady state is defined as:

$$
\|e\|_{L_{2}}=\frac{\left[\sum_{i=1}^{N N}\left(\|u\|_{i}^{n+1}-\|u\|_{i}^{n}\right)^{2}\right]^{1 / 2}}{\left[\sum_{i=1}^{N N}\left(\|u\|_{i}^{n+1}\right)\right]^{1 / 2}}
$$

where $N N$ is the number of nodes in the mesh.
Whatever the method of discretization used to solve the governing equations (transport or the Navier-Stokes equations), the resulting system of algebraic equations can be organized in the following matrix form:

$$
K \Phi=F
$$

in which the global matrix $K$ is composed of the contribution of all finite element matrices, $\Phi$ is the global vector of all degrees of freedom of the problem and $F$ is the global vector of the known terms.

The matrix $K$ is constructed in an assembly process in the following way:

$$
K=\sum_{i=1}^{\text {nelem }} K^{e}
$$

where $K^{e}$ are the element matrices and nelem is the total number of elements. The vector $F$ is constructed similarly. In general, the matrix $K$ is very large and sparse (over $99 \%$ of its elements are null). In this sense, techniques that take into account the sparsity of the matrix make the solution process more efficient.

\section{NUMERICAL RESULTS INCOMPRESSIBLE FLOWS}

In this work, three tests were done using the semi-implicit algorithm CBS (the pressure is obtained from the Poisson equation) in the numerical simulations of Navier-Stokes problems. In the first case the flow with heat transfer was simulated in a channel with an obstacle near the entrance. In the second and third cases, the flows through banks of tubes were simulated.

Linear triangular element meshes were used, since this type of element has a lower computational cost and there are several mesh generators.

In the case of semi-implicit CBS scheme, the Poisson equation for pressure is solved by the conjugate gradient method with Jacobi preconditioner. In other words, the diagonal elements of the matrix are used as pre-conditioner.

\section{Flow in a Square Cavity with a Sliding Wall}

The classic case of a flow in a square cavity induced by movement of the upper wall was solved using the CBS scheme. Figure 2 shows the results for the velocity component $\mathrm{U}$ as a function of $\mathrm{Y}$ for $\mathrm{X}=$ 0.5 , at different times, compared with the results of Ghia et al. (1982), for Reynolds number equal to 1000. Figure 3 shows the results for the velocity component $\mathrm{V}$ a function from $\mathrm{X}$ for $\mathrm{Y}=0.5$. 
One can observe that the present results are in good agreement with the results of Ghia et al. (1982), which validated the CBS scheme.

\section{Flow in a Channel with an Obstacle}

In this case, the development of velocity field was simulated in a channel with the following dimensions: $0 \leq x \leq 12.375$ and $0 \leq y \leq 2$.

An obstacle of square section is located at $4 \leq x \leq 4.5$ and $0.75 \leq y \leq 1.25$.

The mesh in this case has 2169 nodes and 4060 elements. The number of Prantdl was set at 0.72 and different Reynolds numbers was investigated. The velocity boundary condition at the channel and the obstacle walls was set equal to zero (no slip condition).

A parabolic velocity profile was imposed at the entrance of the channel:

$$
u=6 \bar{u}\left(\frac{y}{2 H}-\frac{y^{2}}{2 H^{2}}\right)
$$

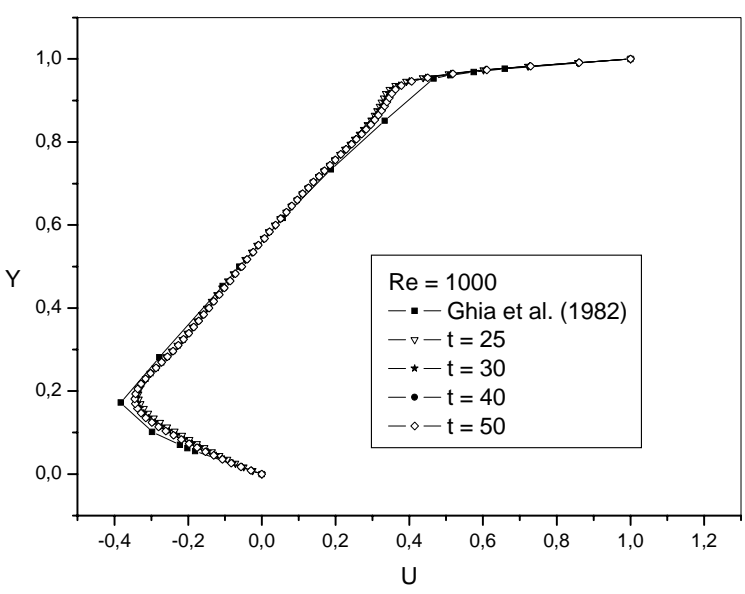

Figure 2. Profile of velocity $\mathrm{U}$ in a cavity with sliding wall, $\mathrm{X}=0.5$.

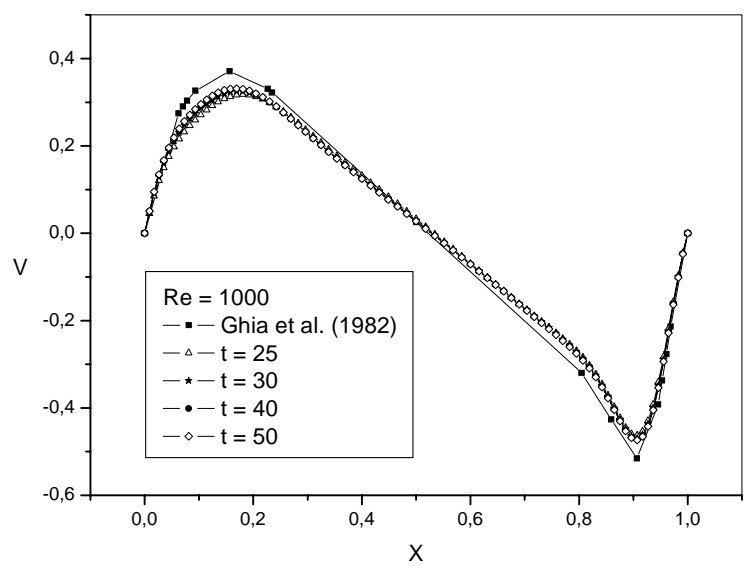

Figure 3. Profile of velocity $\mathrm{V}$ in a cavity with sliding wall, $\mathrm{Y}=0.5$.
The temperature boundary condition at the entrance of the channel was set equal to zero and at the obstacle was set equal to one. In the channel output the pressure boundary condition was set equal to zero.

Figures 4 and 5 show the numerical results for the Reynolds number of 100 , for three different time steps. Figure 4 shows the variation of the temperature field with time. Initially there is a more heated region only around the obstacle.

Over time this region reaches the exit of the channel. Figure 5 presents the path lines, showing the recirculation region behind the obstacle.

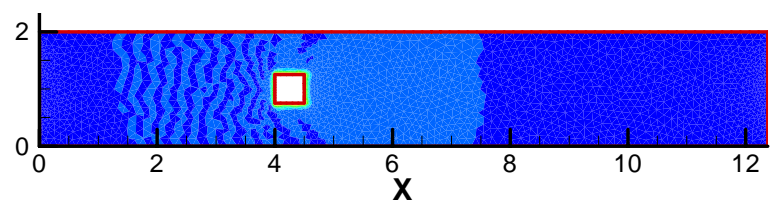

(a)

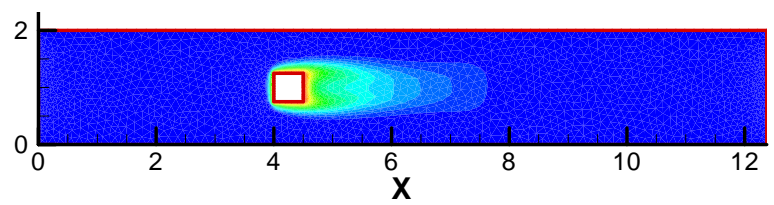

(b)

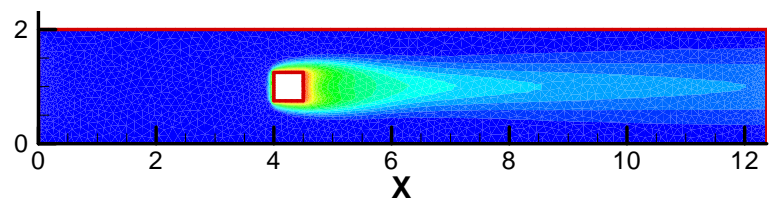

(c)

Figure 4. Temperature field $(\operatorname{Re}=100)$ for the dimensionless time (a) 1, (b) 30 and (c) 75.

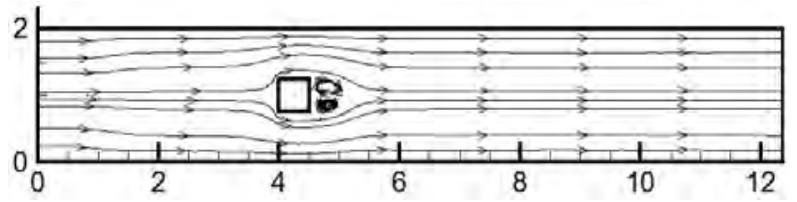

(a)

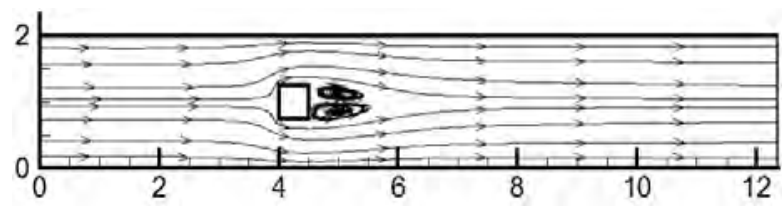

(b)

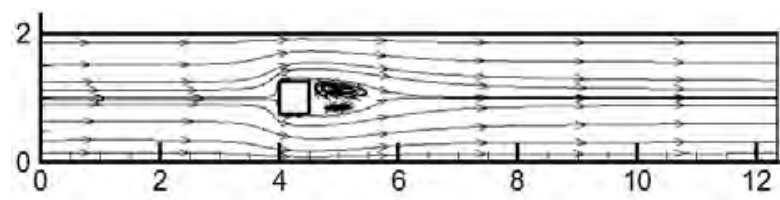

(c)

Figure 5. Streamlines $(\mathrm{Re}=100)$ for the dimensionless time (a) 1, (b) 30 and (c) 75. 
Figures 6 and 7 present the numerical results for the Reynolds number equal to 800 , for steady state flow. Note that the velocity profile at the channel output in steady state is very close to a parabolic profile. The temperature field follows the same behavior of $\operatorname{Re}=100$.

The numerical results for Reynolds number equal to 1200 are shown in Figs. 8 and 9. The velocity field behavior is similar to the results for Reynolds number equal to 800 , but the velocity profile at the exit of the channel is a little flatter in the center, probably due to the undeveloped flow at the channel exit. For a larger number of Reynolds, even for laminar flow, the channel length to achieve a developed profile is greater. Streamlines for $\mathrm{Re}=$ 1200 show that the vortex wake extends to the exit of the channel.

\section{Flow in Staggered Tube Bank}

Bank tubes are commonly found in heat exchangers, so the flows in this type of geometry are of great interest. A staggered bank tube is illustrated

in Fig. 10, where we consider only the domain in which the flow repeats itself periodically.

In this case it is considered only the geometry in the region $(0<x<1.5$ and $0<y<1)$ shown in Fig. 11 . The numerical mesh has 5864 nodes and 11,420 elements. The Prantdl number was set at 0.72 and were considered different Reynolds numbers in the simulations.

The boundary conditions were specified as follows: at the inlet $u=1 ; v=0 ; T=0$; at the outlet $p=0$; at the horizontal lines (symmetry condition) $\frac{\partial u}{\partial y}=0, \frac{\partial T}{\partial y}=0, v=0$ at the tube surfaces $u=0$; $v=0 ; T=1$.

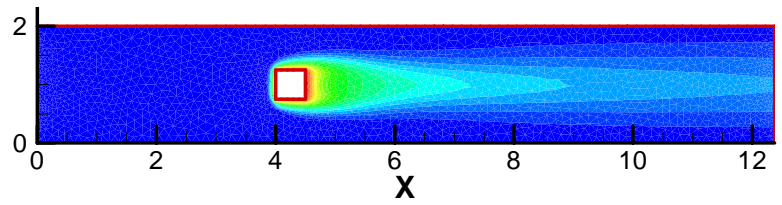

Figure 6. Temperature field $(\mathrm{Re}=800)$ in steady state flow.

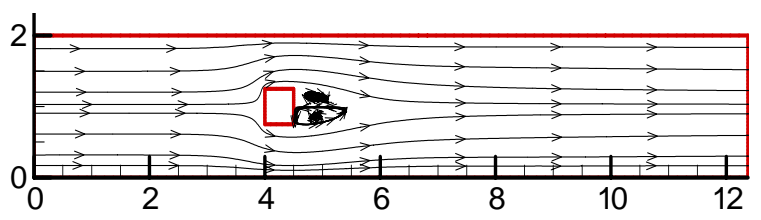

Figure 7. Streamlines $(\mathrm{Re}=800)$ in steady state flow.

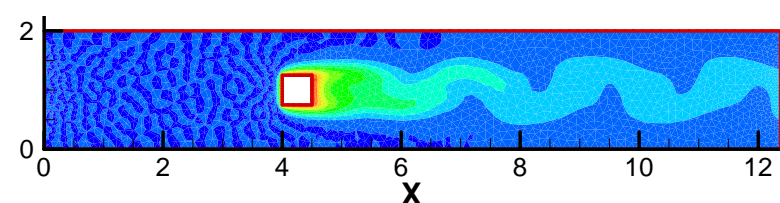

(a)

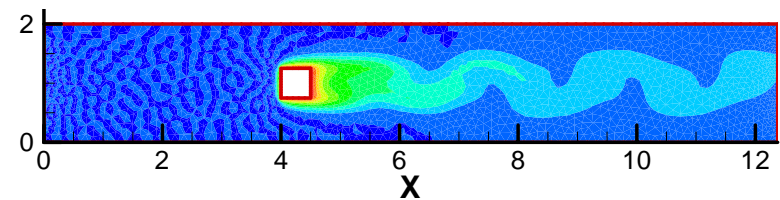

(b)

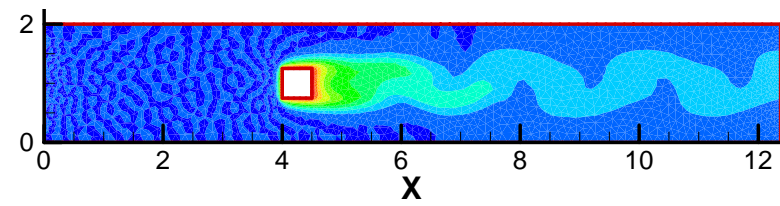

(c)

Figure 8. Temperature field $(\mathrm{Re}=1200)$ for the dimensionless time (a) 1, (b) 4 and (c) 6 .

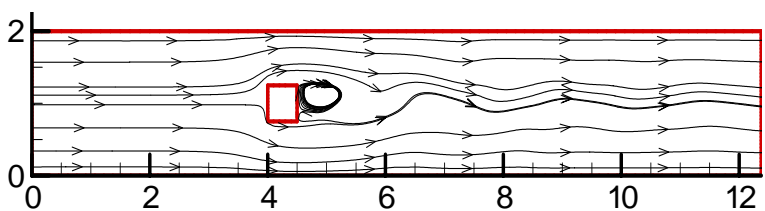

(a)

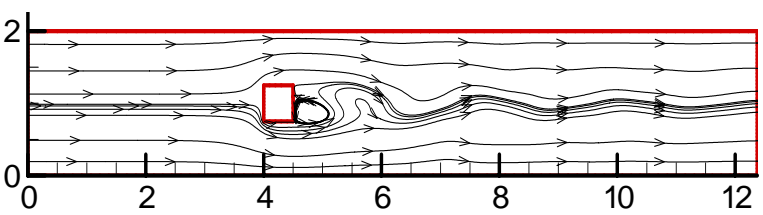

(b)

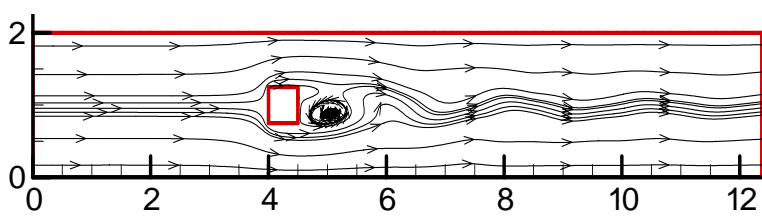

(c)

Figure 9. Streamlines $(\mathrm{Re}=1200)$ for the dimensionless time (a) 1, (b) 4 and (c) 6 .

A zero velocity field was used as initial condition, except at the inlet and the tube surfaces.

Figures 12 and 13 present the numerical results, as a function of the dimensionless time, for Reynolds numbers equal to 100 . It is observed that the the qualitative behavior of the flow between tubes was obtained properly. Figure 12 shows that the behavior of the velocity field for this Reynolds number does not change significantly for the different time steps. However, Fig. 13 shows that the temperature field undergoes major changes during the same time steps. 


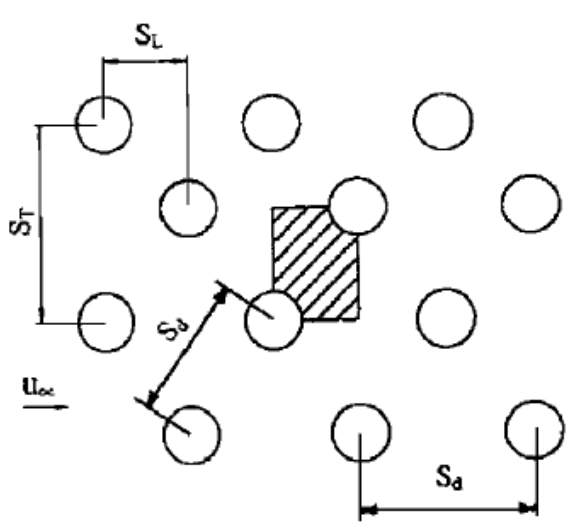

Figure 10. Staggered bank tubes and the numerical domain.

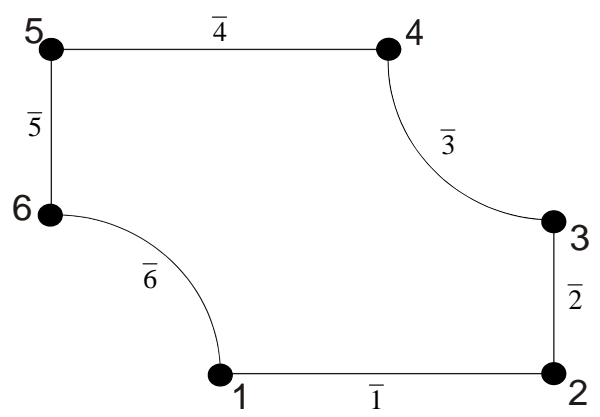

Figure 11. Geometry considered in the numerical simulations.

Simulation results for Reynolds numbers equal to 600 and 1000 are presented in Figs. 15 to 18. It can be observed that the length of the recirculation region behind the first cylinder increases with increasing Reynolds number, as can be seen in Figs. 15 and 17. The temperatures fields shown in Figs. 16 and 18 behave consistent with the boundary conditions imposed for this geometry.

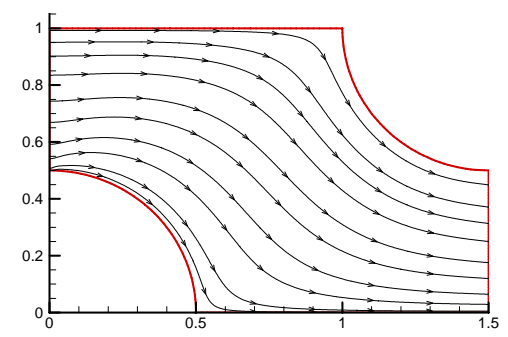

(a)

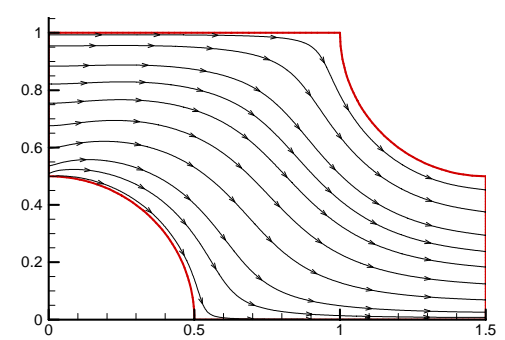

(b)

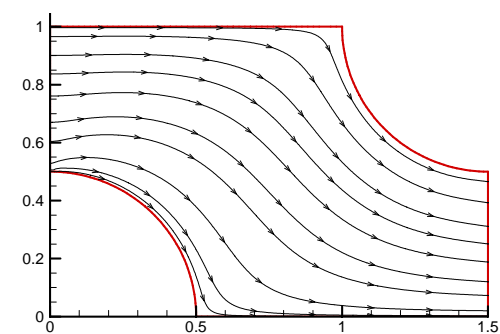

(c)

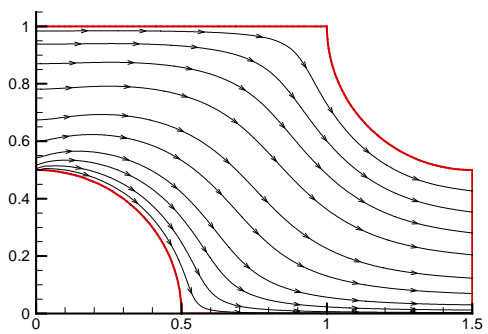

(d)

Figure 12. Stream lines $(\operatorname{Re}=100)$ for dimensionless time steps (a) 1, (b) 4, (c) 8 and (d) 14.

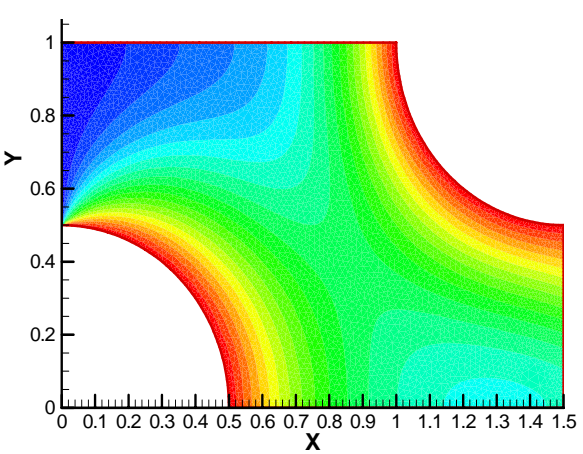

(a)

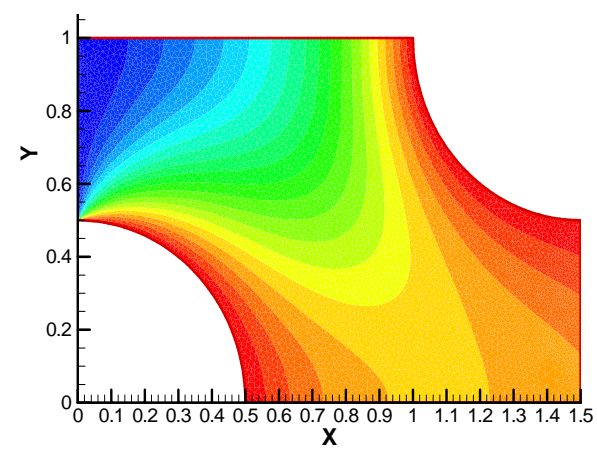

(b)

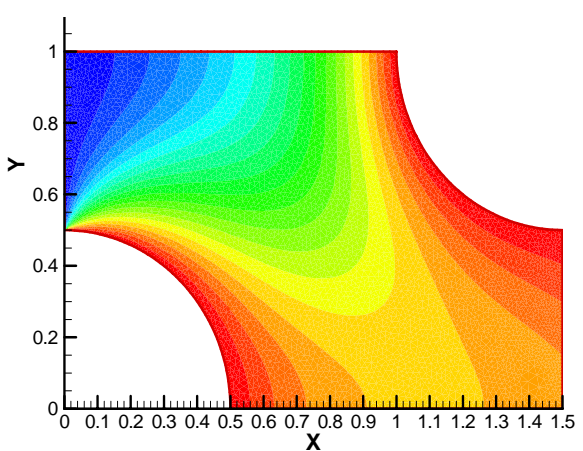

(c) 


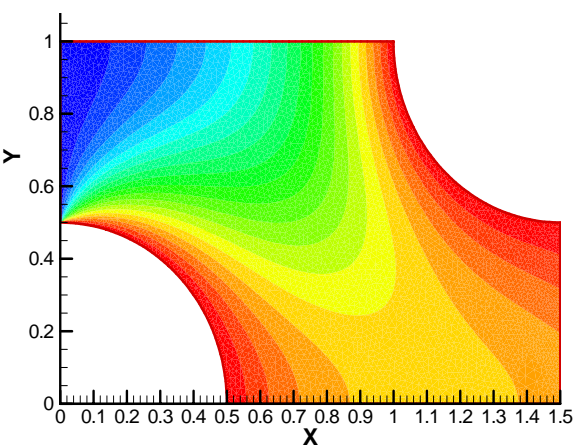

(d)

Figure 13. Temperature field $(\mathrm{Re}=100)$ for time steps (a) 1, (b) 4, (e) 8 and (f) 14.

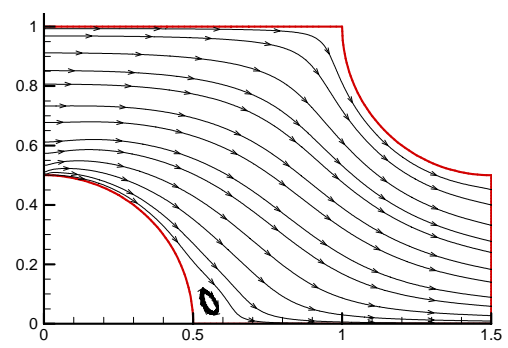

(a)

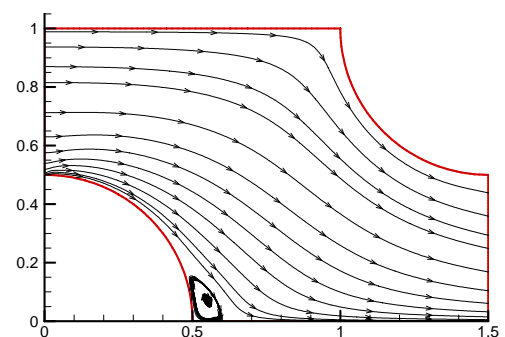

(b)

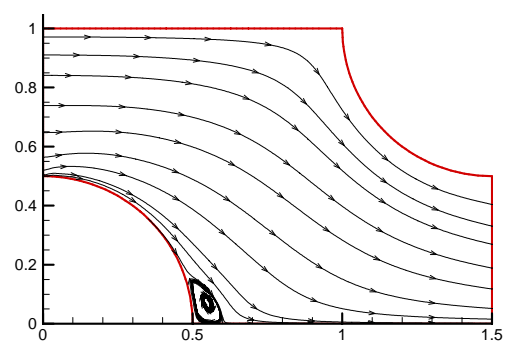

(c)

Figure 14. Stream lines $(\operatorname{Re}=600)$ for time steps (a) 1 , (b) 3 and (c) 5 .

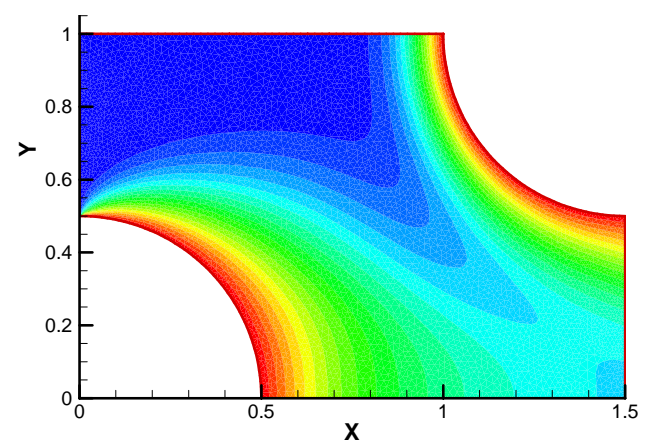

(a)

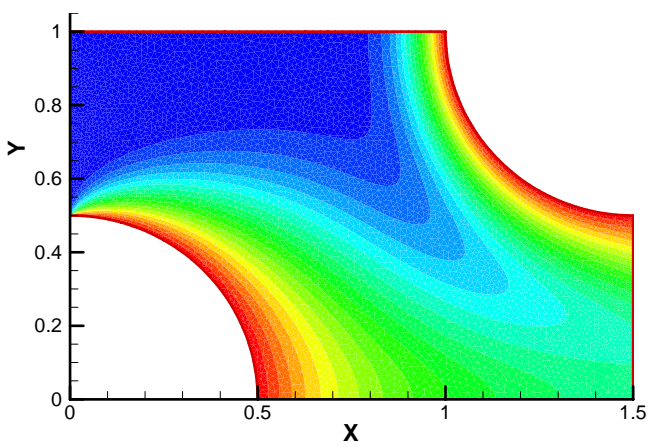

(b)

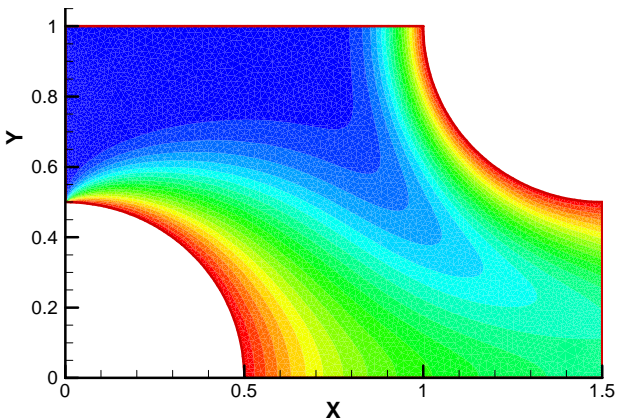

(c)

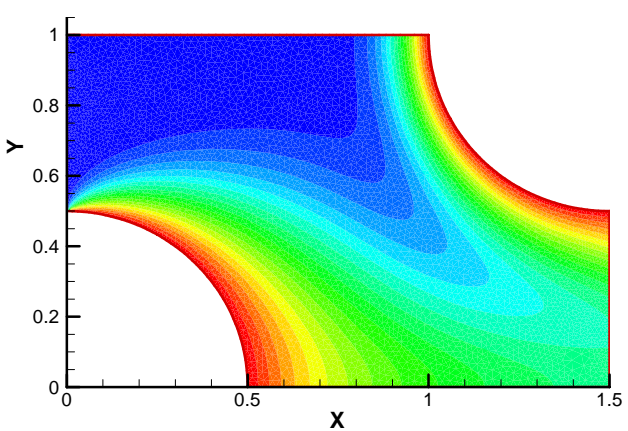

(d)

Figure 15. Temperature field $(\mathrm{Re}=600)$ for time steps (a) 1, (b) 2, (c) 5 and (d) 7.

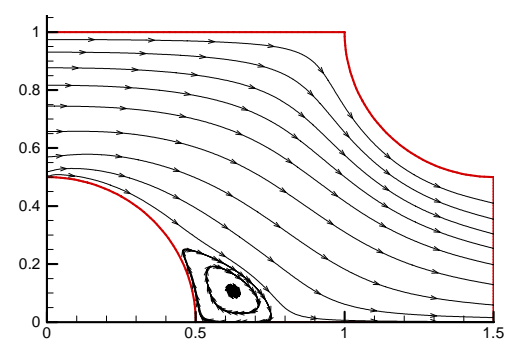

(a)

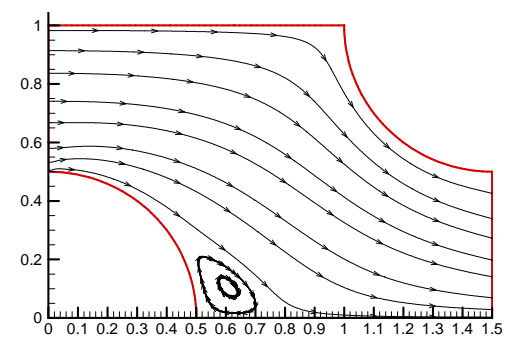

(b) 


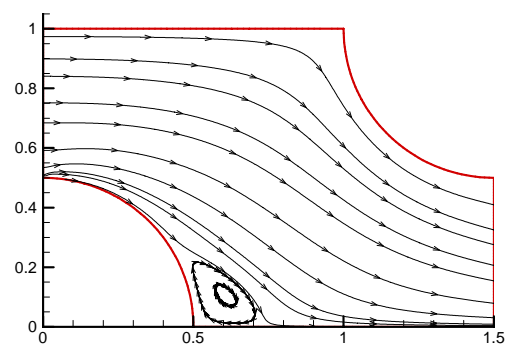

(c)

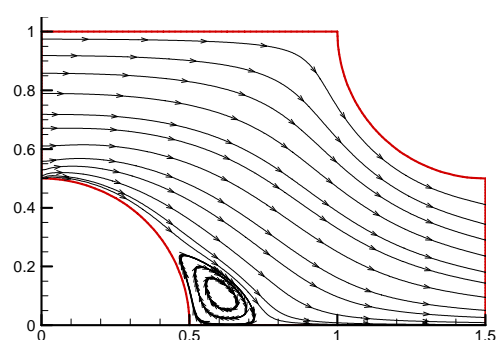

(d)

Figure 16. Stream lines $(\operatorname{Re}=1000)$ for time steps $(a)$ 1, (b) 4, (c) 6 and (d) 8 .

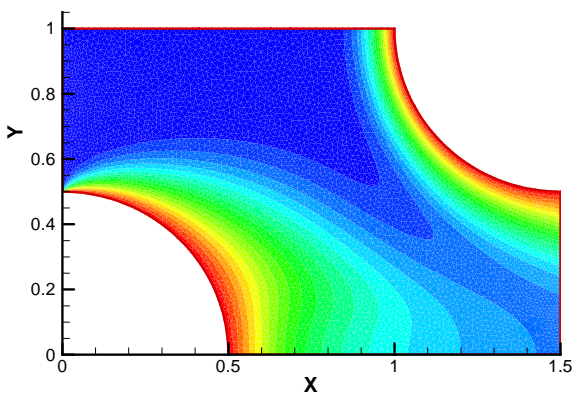

(a)

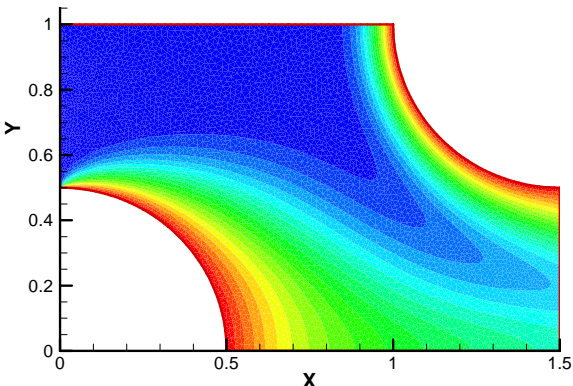

(b)

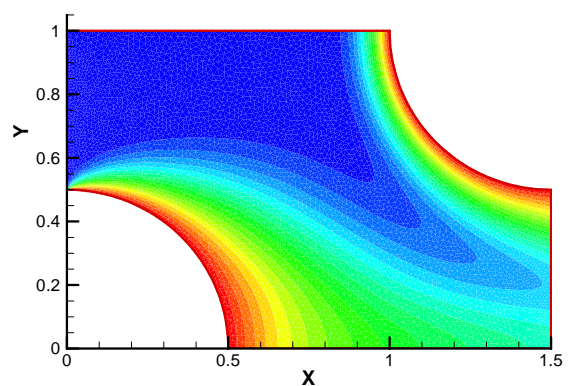

(c)

Figure 17. Temperature field $(\mathrm{Re}=1000)$ for time steps (a) 1 , (b) 5 and (c) 8 .

\section{Flow in an Aligned Tube Bank}

The geometry of the aligned tube bank, shown in Fig. 18, represents the flow in the wake of one tube. The physical domain was defined in the region: $0 \leq x \leq 10$ and $0 \leq y \leq 2$.

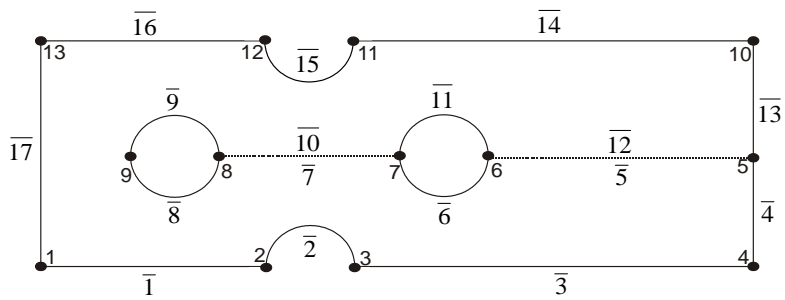

Figure 18. Geometry of the aligned tubes bank.

In this case, a uniform velocity profile was specified at the entrance area. The boundary conditions were imposed as follows: at the inlet $u=$ $1, v=0, T=0$; at the outlet $p=0$; symmetry condition on the horizontal lines, $\partial u / \partial y=0, \partial T / \partial y=$ $0, v=0$ and no slip condition and unitary temperature on the tube walls, $u=0, v=0, T=1$.

Figures 19 and 21 present the time evolution of the temperature field for $\operatorname{Re}=100$ and $\operatorname{Re}=400$, respectively. Thses numerical results show that the heat transfer process it consistently simulated. By inspection of Fig. 20, it can be seen that the presence of a second cylinder on the wake of the first one dampens the recirculation behind the first cylinder. It may be noted in Figs. 21 and 22 that for a larger Reynolds number, the recirculation region behind the second cylinder increases as expected.

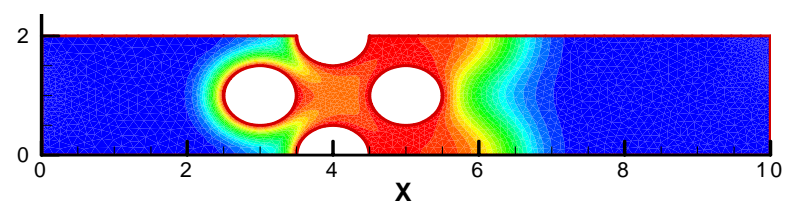

(a)

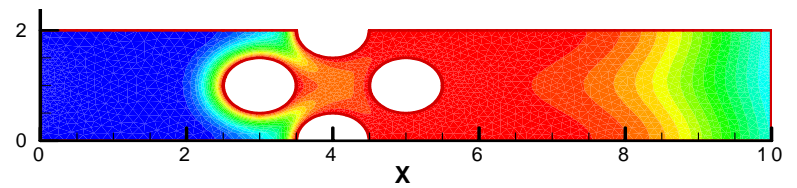

(b)

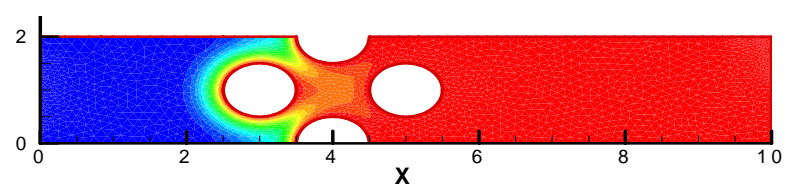

(c)

Figure 19. Temperature field $(\mathrm{Re}=100)$ for time steps (a) 1, (b) 4 and (c) 28. 


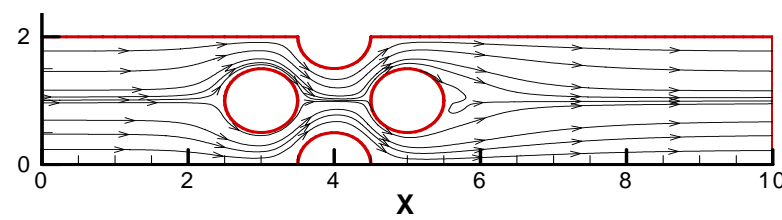

(a)

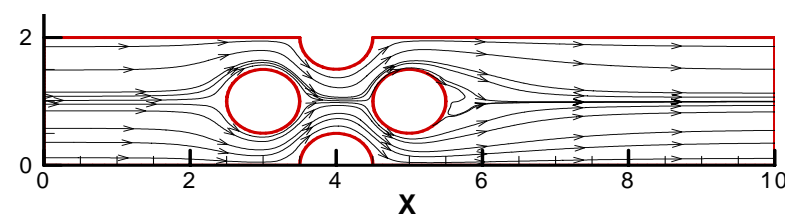

(b)

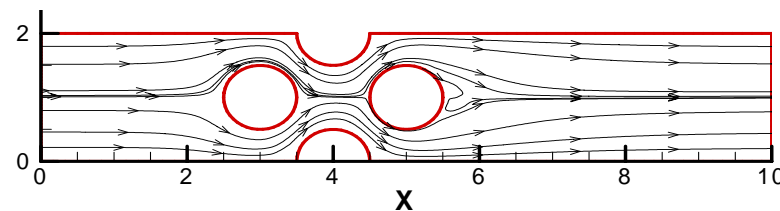

(c)

Figure 20. Stream lines $(\mathrm{Re}=100)$ for time steps (a) 1 , (b) 4 and (c) 28 .

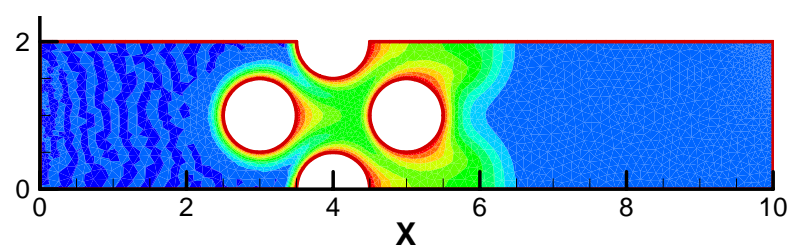

(a)

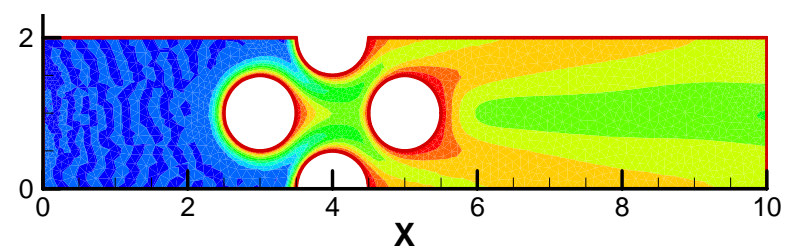

(b)

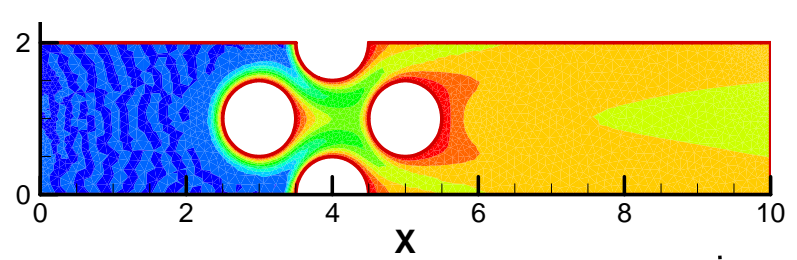

(c)

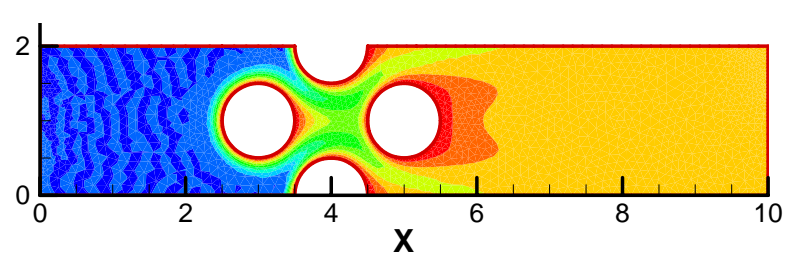

(d)

Figure 21. Temperature field $(\mathrm{Re}=400)$ for time steps (a) 1, (b) 6, (c) 11 and (d) 21.

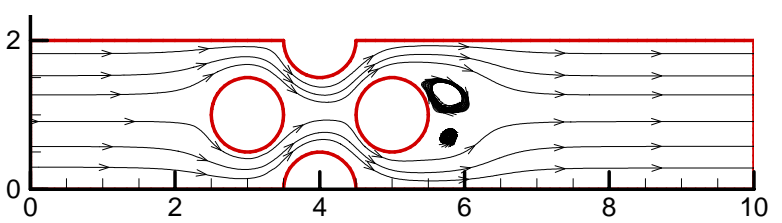

(a)

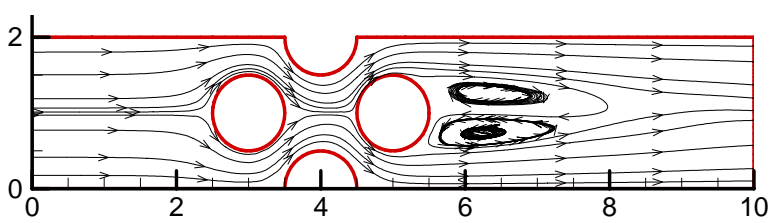

(b)

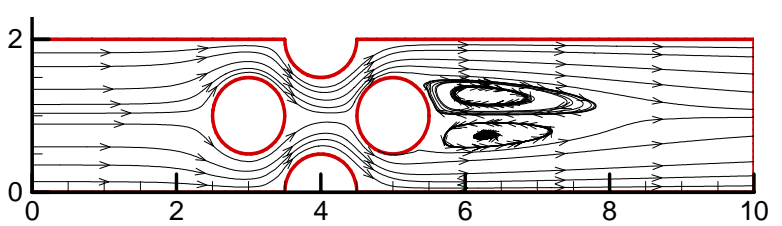

(c)

Figure 22. Stream lines $(\mathrm{Re}=400)$ for time steps $(\mathrm{a}) 1$, (b) 11 and (c) 21 .

\section{CONCLUSIONS}

In this work a stabilization method, called Characteristic Based Scheme (CBS), was applied to the numerical solution of the two-dimensional Navier-Stokes equations and the transport equation for the temperature field. In the numerical applications, the physical domains were discretized by using unstructured meshes of linear triangular elements, that adapt well to any geometry and has a lower computational cost compared to other types of elements. Unstructured meshes also allow the refining regions of interest without the need to refine the entire domain.

The computational program developed in this work was applied to the numerical solution of incompressible flows in a channel with an obstacle and in staggered and aligned tube banks, for different Reynolds numbers. The CBS proved to be an effective tool in solving complex problems, encouraging its application to other flow problems.

\section{REFERENCES}

Ghia U, Ghia K. N., and Shin, C. T., 1982, High-Re Solutions for Incompressible Flow Using the Navier-Stokes Equations and a Multigrid Method, Journal of Computational Physics, Vol. 48, pp. 387411.

Jiang, B. N., 1998, The Least-Squares Finite Element Method: Theory and Applications in Computational Fluid Dynamics and Electromagnetics, Springer.

Lewis, R. W., Nithiarasu, P., and Seetharamu, K. N., 2004, Fundamentals of the Finite Element Method for Heat and Fluid Flow, John Wiley. 
Liu, C. B., 2005, The Characteristic Based Split (CBS) Sheme for Laminar and Turbulent Incompressible Flow Simulations, Doctoral Thesis, University of Wales Swansea, Wales, United Kingdom.

Reddy, J. N., 1993, An Introduction to the Finite Element Method, Second Edition, McGraw-Hill.

Zienkiewicz, O. C., Taylor, R. L., and Nithiarasu, E. P., 2005, The Finite Element Method for Fluid Dynamics, Elsevier. 